\title{
La cirugía de epilepsia y el establecimiento de programas quirúrgicos en el Perú: El proyecto de colaboración entre Perú y Canadá.
}

Establishing epilepsy surgery programs in Peru: The Perú-Canada collaborative project.

Jorge G Burneo ${ }^{1}$, David A Steven ${ }^{1}$, Miguel Arango ${ }^{2}$, Willy Zapata ${ }^{3}$, Carlos M Vasquez ${ }^{3}$, Alicia Becerra ${ }^{4}$

\section{RESUMEN}

El presente artículo define y describe las características clínicas de epilepsia refractaria, la cirugía aplicable al manejo de la misma y la necesidad de establecer centros para el manejo integral de estos pacientes en el Perú. Además, describe los inicios de la cirugía de epilepsia en el Perú, su desarrollo paulatino y el proyecto de colaboración asistencial y educativo entre los programas de epilepsia de la Western University en London, Ontario, Canadá y el Instituto Nacional de Ciencias Neurológicas, así como el Hospital Edgardo Rebagliati en Lima, Perú.

PALABRAS CLAVE: Epilepsia, cirugía de epilepsia, lobectomía, temporal, Perú, Canadá.

\section{SUMMARY}

The manuscript describes and defines the clinical characteristics of Refractory Epilepsy, applicable surgical procedures for its management and the need for the establishment of comprehensive epilepsy programs in Peru. It also describes the beginnings of epilepsy surgery in Peru, and the collaborative project between the Epilepsy programs at Western University in London, Canada and the National Neurological Institute and Rebagliati Hospital in Lima, Peru.

KEYWORDS: Epilepsy, epilepsy surgery, lobectomy, temporal, Peru, Canada.

\section{INTRODUCCIÓN}

La epilepsia representa un problema de salud pública, ya que es la segunda condición neurológica más frecuente a nivel mundial y constituye la condición neurológica con mayores perjuicios a nivel mundial por incapacidad, mortalidad, comorbilidad, estigma y costos (1). Si bien la prevalencia en los países del hemisferio norte está entre 5-6 por cada
1000 personas, en Latino América es más alta. En el Perú, se estima que la prevalencia de epilepsia es de 11,9-32,1 por cada 1000 personas (2).

La mayor parte de pacientes con epilepsia responde al tratamiento con fármacos antiepilépticos (FAEs), sin embargo, alrededor de un $30 \%$ presentan una situación contraria. En estos casos se debe considerar como tratamiento, la cirugía de epilepsia (3).

\footnotetext{
1 Epilepsy Program, Department of Clinical Neurological Sciences, Schulich School of Medicine, Western University. London, Canada.

2 Anesthesia \& Perioperative Medicine, Schulich School of Medicine, Western University, London Health Sciences Center. London, Canada.

3 Departamento de Epilepsia, Instituto Nacional de Ciencias Neurológicas. Lima, Perú.

4 Departamento de Neurocirugía, Hospital Edgardo Rebagliati Martins. Lima, Perú.
} 
Si bien la cirugía de epilepsia existe desde la última década de los años 1800 , esta se volvió más común entre 1970 y 1980. Lamentablemente, muchos países del mundo aun no cuentan con este tipo de tratamiento. En Latinoamérica, con excepción de Brasil y Colombia, y ahora Perú, los centros de cirugía de epilepsia se encuentran en centros médicos $\mathrm{u}$ hospitales privados, haciéndola no disponible para gran proporción de la población general.

El proyecto de colaboración entre Perú y Canadá nació en el 2008 con la intención de establecer programas de cirugía de epilepsia en el Perú (4). El presente documento describe el proyecto desde sus albores hasta la actualidad

\section{Cirugía de epilepsia}

\section{¿Qué son la epilepsia refractaria y la cirugía de epilepsia?}

En epilepsias de tipo focal, cerca del $50 \%$ de pacientes responden apropiadamente al primer tratamiento adecuado con FAEs. Un 15-20\% responde al segundo tratamiento adecuado. Luego de eso, solo menos del 5\% de pacientes responderán al tratamiento con FAEs. Cuando la epilepsia no responde al tratamiento adecuado, por un periodo menor a tres veces el tiempo de duración del mayor intervalo de tiempo sin crisis, con por lo menos 2 FAEs, se le conoce como epilepsia refractaria $(3,5)$. En el caso de las epilepsias generalizadas, la refractariedad se aprecia en aproximadamente el $20 \%$ de ellas (6).

En base a los resultados del último censo peruano realizado por el Instituto Nacional de Estadística e Informática (INEI) el año 2014, en el cual se indica que la población del Perú es de aproximadamente 31 millones de habitantes, la población con epilepsia podría estar cercana al medio millón de personas, de las cuales muy probablemente 150 mil tienen epilepsia refractaria.Lamentablemente, no se sabe con certeza el número de pacientes con epilepsia refractaria, ya que no existen estudios epidemiológicos nacionales al respecto.

Cuando se hace el diagnóstico de epilepsia refractaria, se tiene que considerar otras formas de tratamiento, las cuales incluyen terapias con dietas especiales y la cirugía de epilepsia $(7,8)$.

El principal objetivo de la cirugía de epilepsia, en aquellos pacientes con epilepsia de tipo focal, es la remoción del foco epileptogénico de la corteza cerebral, con tasas de remisión de crisis epilépticas de aproximadamente $64 \%$ en epilepsia del lóbulo temporal (7), y 36-76\% en aquellos con epilepsia extratemporal (9). En algunos casos, son lesiones las

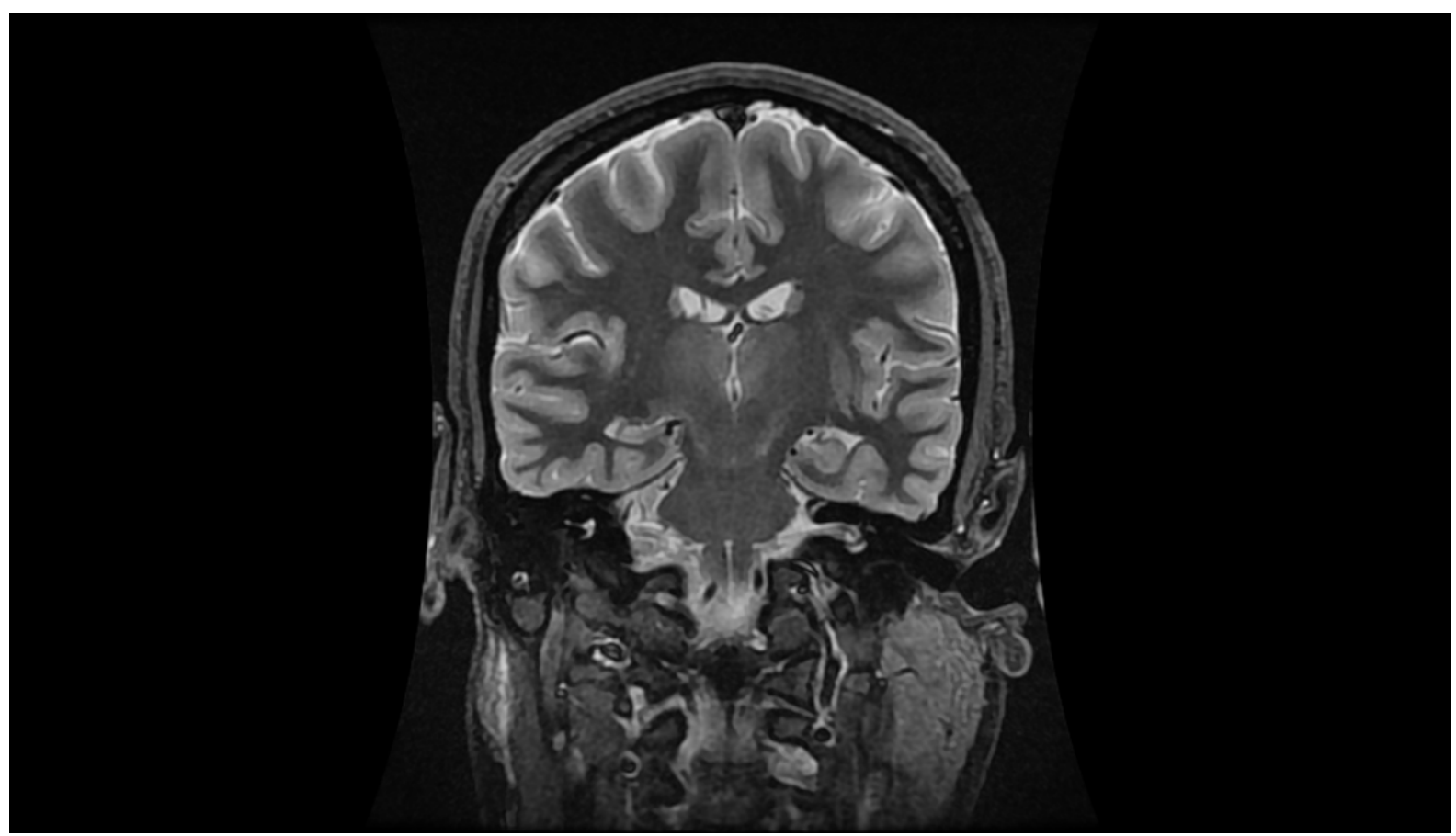

Figura 1. Resonancia magnética cerebral con protocolo de epilepsia, en sección coronal, secuencia T1 de alta resolución (Imagen proporcionada por el Programa de Epilepsia de la Western University, London, Canada) 


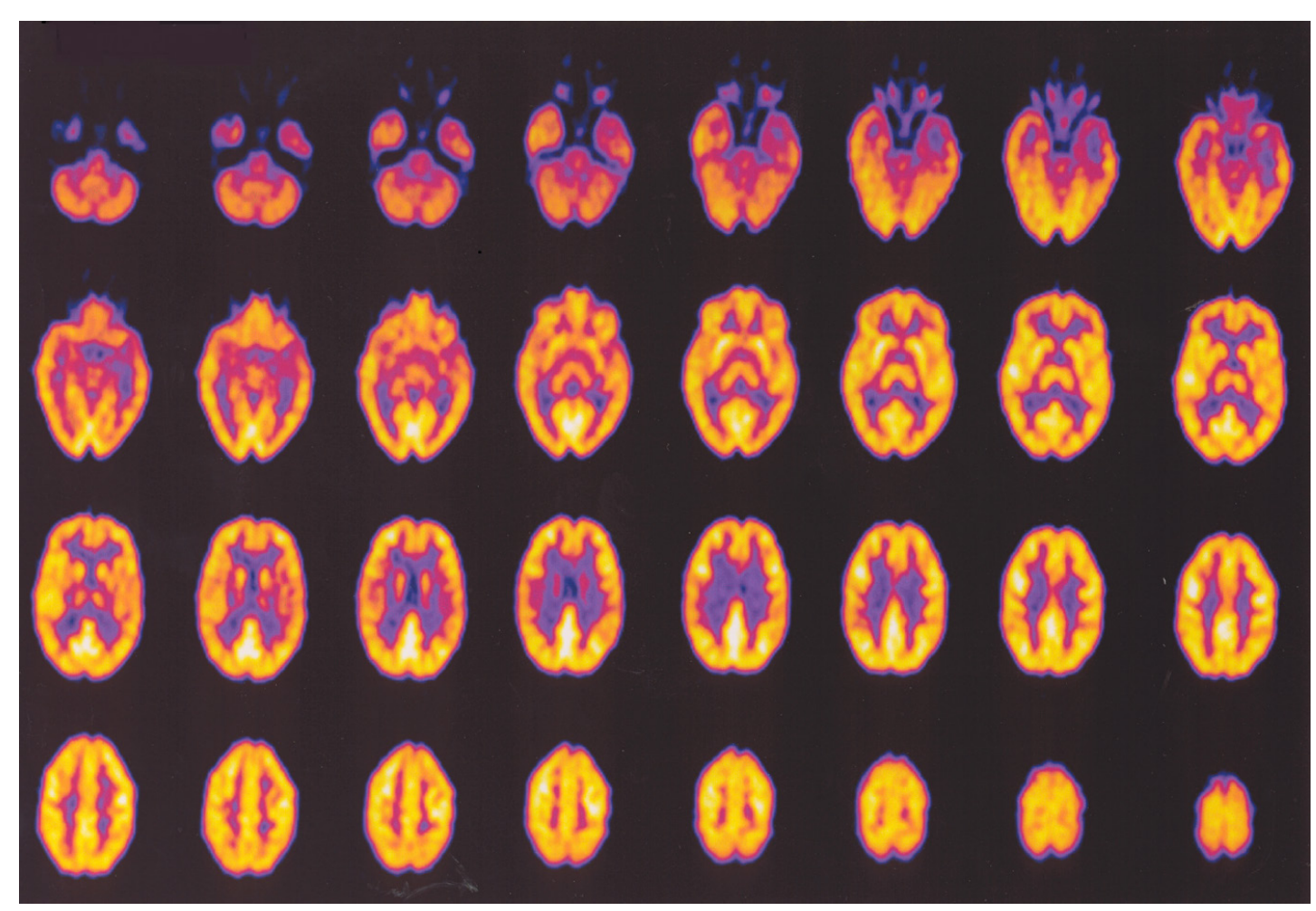

Figura 2. Tomografía por emisión de positrones. En este caso existe hipo-metabolismo mesial temporal izquierdo (Imagen proporcionada por el Programa de Epilepsia de la Western University, London, Canada).

que originan crisis epilépticas, pero en alrededor del $30 \%$ de los casos, no existe lesión alguna identificada con estudios de resonancia magnética (RM) cerebral; a pesar de esto último, la cirugía resectiva de epilepsia está indicada siempre y cuando se pueda ubicar el foco epileptogénico mediante una serie de investigaciones conocida como evaluación prequirúrgica. En los casos en los cuales no existe un foco, sino la epilepsia es más regional o hemisférica, así como en el caso de las epilepsias generalizadas, las cirugías son consideradas paliativas e incluyen la hemisferotomía y la callostomía. Finalmente, existe también la neuromodulación en donde se incluyen la estimulación del nervio vago, la estimulación de los núcleos anteriores de ambos tálamos, la estimulación trigémina, y la estimulación responsiva de corteza. La neuromodulación tiene como objetivo principal reducir las crisis, sin embargo, en algunos casos puede llegar a abolir las crisis completamente (10).

Antes del año 2011, la cirugía de epilepsia en el Perú no existía, con la excepción de cirugías ocasionales, que consistían en remociones de tumores, malformaciones arterio-venosas y otras lesiones estructurales que no necesariamente tenían como objetivo ayudar al control de las crisis, sin embargo, no existían programas integrales de cirugía de epilepsia de ningún tipo y menos aún, con algún equipo multidisciplinario especializado.

\section{¿En qué consiste un programa de cirugía de epilepsia?}

Los programas integrales de cirugía de epilepsia se encargan de evaluar al paciente con epilepsia refractaria. Su finalidad es la de identificar si el paciente con epilepsia refractaria es candidato para algún tipo de cirugía de epilepsia. Para poder llevar a cabo una cirugía de epilepsia existen requerimientos humanos y de infraestructura que se describen a continuación.

El equipo humano consiste en un equipo multidisciplinario el cual es co-dirigido por un neurólogo y un neurocirujano. Tanto el neurólogo como el neurocirujano requieren de entrenamiento especial en cirugía de epilepsia, el cual se puede adquirir en ciertos centros médicos, con una duración promedio de 1 a 2 años; el neurólogo se gradúa como epileptólogo y el neurocirujano como cirujano de epilepsia, ambos obtienen certificación del centro en 
el cual se entrenaron. En ciertos países o regiones, como en EEUU y en la Unión Europea, se reconoce el entrenamiento en epilepsia como una especialidad de neurología, a la que se le llama epileptología, y como parte del entrenamiento el neurólogo debe tomar un examen de certificación nacional para poder laborar como epileptólogo. En Canadá, el neurólogo, luego de su entrenamiento tiene que rendir el examen nacional de EEG, para tener la autoridad de informar EEGs.

El programa también debe contar con psicólogo(s) con experiencia en la evaluación de pacientes con problemas psiquiátricos asociados a la epilepsia, como depresión y ansiedad. En algunos centros se cuentan con Neuro-psiquiatras o psiquiatras. Es también importante, la presencia de un neuro-psicólogo(a) encargado de la evaluación cognitiva, del lenguaje y la memoria, con el objetivo de identificar áreas de déficit que coincidan con el foco epileptogénico, además de ayudar a predecir, de cierta manera, los déficits que podrían ocurrir en estas áreas después de una cirugía cerebral. El equipo también debe de contar con un(a) trabajador(a) social, farmacéutico(a), enfermeras y tecnólogas de electroencefalografía.
En centros de referencia mundial en el manejo de la cirugía de epilepsia existe además la presencia de anestesiólogos con interés en neuro-anestesia. Procedimientos como la craneotomía despierta necesitan de personal quirúrgico altamente entrenado para lograr resultados intra-operatorios adecuados.

En cuanto al requerimiento de infraestructura, este se refiere a la necesidad de contar con camas hospitalarias destinadas a la realización de estudios de video-EEG, idealmente por más de 24 horas. Además, se requiere de equipos de video-EEG con un hardware y software que permita el registro por 24 horas y el almacenamiento de la información recabada.

\section{Evaluación pre-quirúrgica}

Se divide en 2 fases, la primera fase consiste en el monitoreo del paciente con video-EEG continuo. La idea es obtener un registro interictal (entre crisis) y uno ictal (de las crisis). La cámara de video sincronizada con el registro de EEG permitirá la localización del inicio de las crisis. No existe un número de crisis mínimo que se deba registrar, eso queda a la discreción

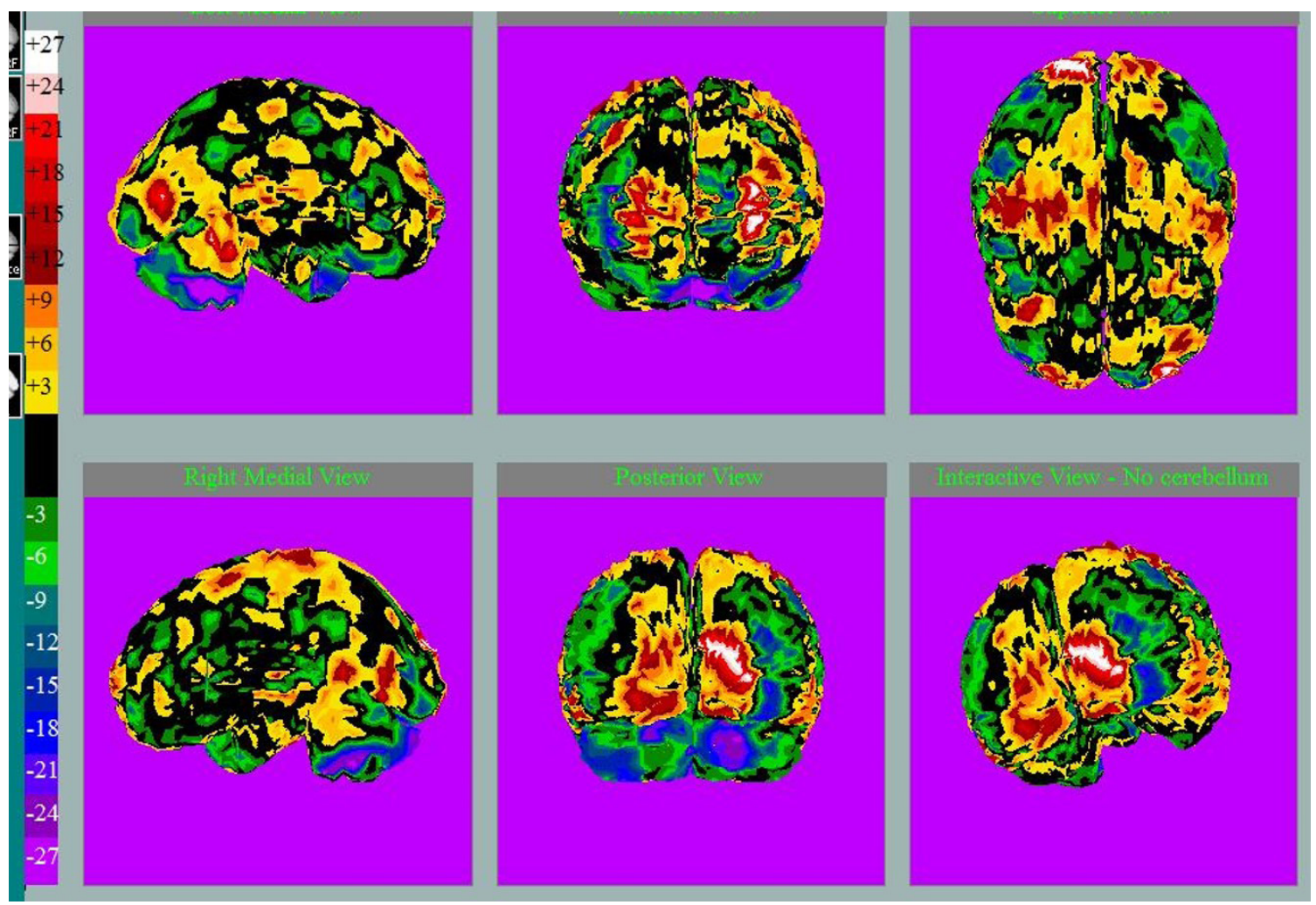

Figura 3. Tomografía por emisión de foton único (SPECT). En esta imagen se aprecia la sustracción de la imagen ictal menos la imagen interictal. La zona blanca representa la zona de mayor hiper-perfusión cerebral. En este caso la zona está en la corteza occipital derecho.(Imagen proporcionada por el Programa de Epilepsia de la Western University, London, Canada) 


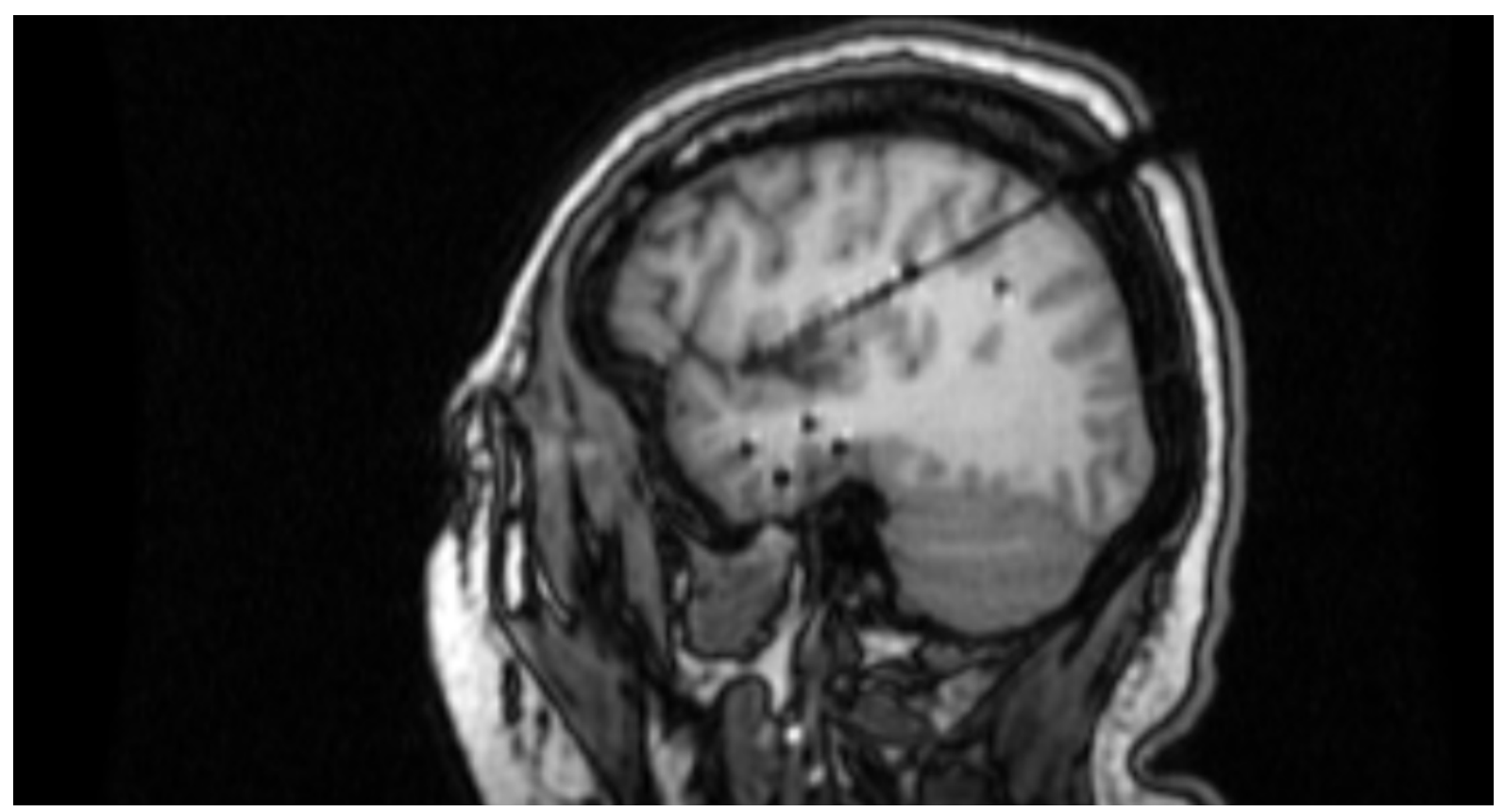

Figura 4. Electrodos intra-craneales profundos (Imagen proporcionada por el Programa de Epilepsia de la Western University, London, Canada).

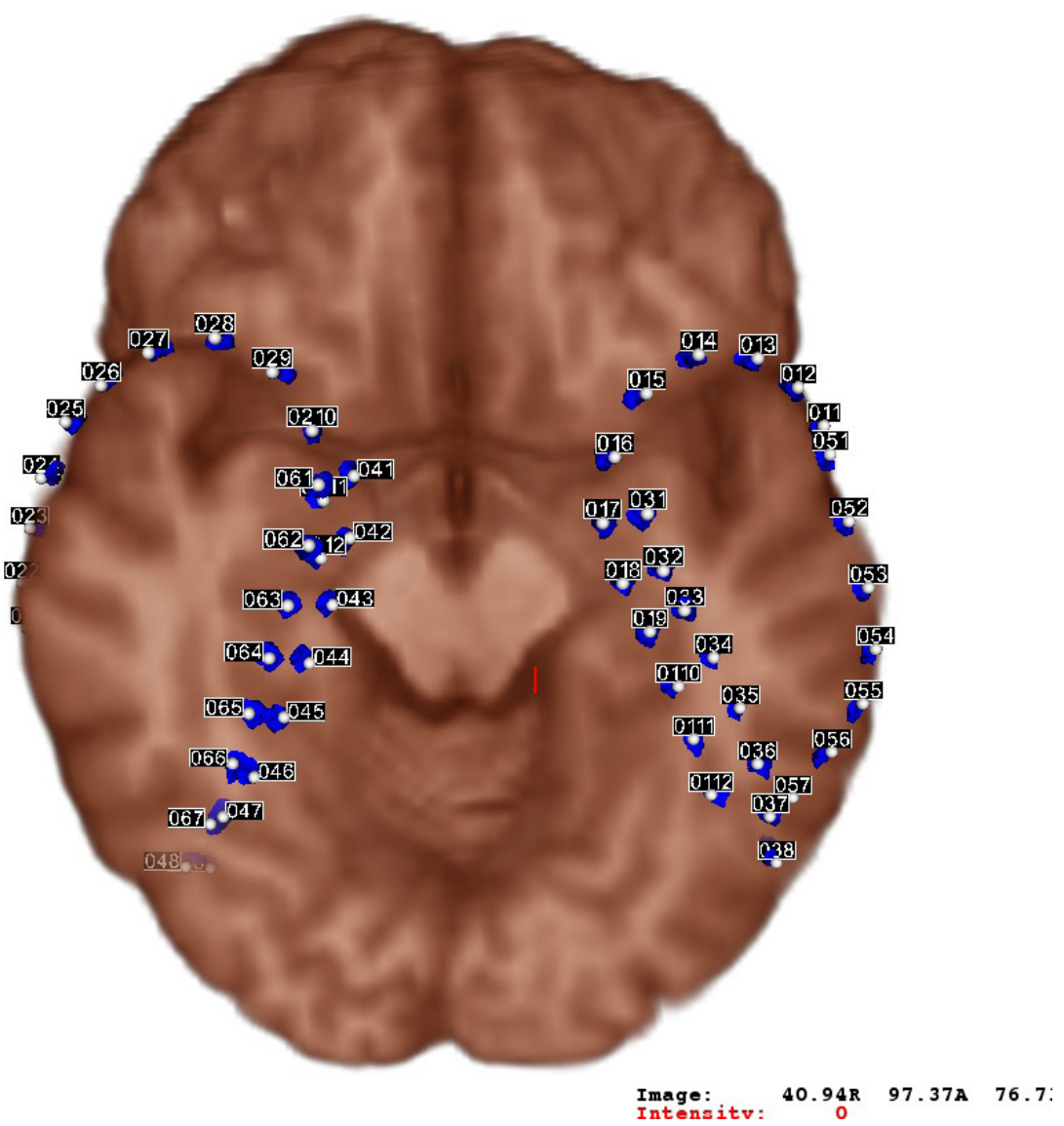

Figura 5. Electrodos intra-craneales subdurales (Imagen proporcionada por el Programa de Epilepsia de la Western University, London, Canada). 
del médico tratante. En muchos centros el promedio fluctúa entre 5 y 7 días de monitoreo continuo con video-EEG. Durante esta hospitalización, se obtiene una RM del cerebro (figura 1), siguiendo protocolos de epilepsia, los cuales incluye cortes a lo largo del eje de los hipocampos, así como cortes coronales finos de los lóbulos temporales y frontales en diferentes secuencias.

Durante la hospitalización para video-EEG o de manera ambulatoria también se realiza una evaluación psicológica y neuro-psicológica. Estas permiten identificar patologías psiquiátricas concomitantes, y sobre todo localización del hemisferio dominante para el lenguaje, así como ciertos déficits que pudieran haber en la memoria verbal y visual, y otras funciones cognitivas, incluyendo atención, coeficiente intelectual, lenguaje, entre otros. Los hallazgos de ciertos déficits indican de manera indirecta la localización del foco epileptogénico, además de dar una idea sobre el posible desenlace neurológico luego de la resección de ciertas partes de la corteza.

Los tres estudios mencionados corresponden a la evaluación mínima que cada paciente tiene que recibir cuando se considera la cirugía de epilepsia. Pero, en algunos centros se realizan estudios de PET y SPECT. El PET o tomografía por emisión de positrones (figura 2 ), los cuales son pruebas inter-ictales que mide de manera cualitativa y cuantitativa el metabolismo de ciertas sustancias, generalmente glucosa, en la corteza cerebral. El hallazgo de una zona hipometabólica, generalmente se correlaciona con el área epileptogénica (11). Por otro lado, el SPECT o tomografía por emisión de fotón único (figura 3), es una prueba ictal e interictal que mide la perfusión cerebral de manera cualitativa y cuantitativa. Se inyecta una sustancia, generalmente con tecnecio (v.g. HMPAO) durante el inicio de una crisis o aura, y se repite cuando el paciente no está teniendo una crisis, de manera que se comparan ambas imágenes con el objetivo de encontrar un área de hipoperfusión cerebral, que se correlacione con el área epileptogénica (12).

En algunos casos, esta evaluación prequirúrgica es suficiente para tomar una decisión sobre si el paciente es candidato o no a cirugía. No sería candidato si la epilepsia es multifocal, o se encuentra en zonas importantes de la corteza, como el lenguaje, región motora y región visual, o si las crisis son generalizadas. En este último caso, las cirugías paliativas estarían indicadas.
Si la información obtenida es completa, se puede proceder a cirugía resectiva o paliativa. Si la información determina que una o ciertas regiones del cerebro pueden estar involucradas, pero que no están determinadas con exactitud, los pacientes pasan a una segunda fase de evaluación, que consiste en la colocación de electrodos intracraneales (figuras 4 y 5), los cuales se conectan a las máquinas de video-EEG para monitoreo prolongado, pero restringido a las zonas donde se encuentran los electrodos. Luego de esta segunda fase, se puede determinar en la mayoría de los casos con exactitud, si el paciente es candidato para cirugía resectiva.

\section{Proyecto colaborativo}

Proyecto colaborativo Perú-Canadá /Ministerio de Salud (MINSA) - Instituto Nacional de Ciencias Neurológicas

En el 2008, la Comisión Norteamericana de la Liga Internacional Contra la Epilepsia (ILAE, siglas en inglés) lanzó un programa llamado PECA (Partnering Epilepsy Programs in the Americas), con la intención de emparejar centros de epilepsia en Norte América y Latinoamérica. El programa de Western University en London (UWO por sus siglas en inglés), Ontario, empezó a colaborar con el Instituto Nacional de Ciencias Neurológicas (INCN) en Lima, gracias a una beca de la ILAE. Esta beca inicialmente permitió la participación del equipo UWO en un taller de video-EEG organizado por el INCN. Luego se llevaron a cabo viajes anuales a Lima por el equipo canadiense, que permitió compartir experiencias en el manejo de pacientes con epilepsia refractaria. De la misma manera, se realizaron viajes de miembros del programa del INCN a la Western University en Canadá para entrenamiento clínico y educación. Además, se realizaron simposios, charlas, conferencias, y rondas en el servicio del INCN. También se desarrollaron de guías de manejo y evaluación del paciente con epilepsia refractaria, con miras a cirugía de epilepsia. Estas guías sirvieron para la organización del programa de cirugía de epilepsia en el INCN. Estas guías fueron creadas simulando el modelo canadiense, pero ajustadas a la realidad hospitalaria peruana. Las visitas del equipo canadiense continuaron de manera continua, cada año, gracias al apoyo financiero de entidades privadas canadienses como Western University y Dominos Pizza Canadá.

La participación conjunta de neurólogos y neurocirujanos canadienses y peruanos, permitió 
La cirugía de epilepsia y el establecimiento de programas quirúrgicos en el Perú.

además entender la importancia del trabajo conjunto de estas dos especialidades, tanto fuera como dentro del quirófano.

En octubre del 2011, se realizó en el Perú, por primera vez una lobectomía temporal en un paciente con epilepsia refractaria del lóbulo temporal. Esta fue llevada a cabo por el neurocirujano de epilepsia del INCN, Dr Carlos Mao Vasquez, quien realizó su entrenamiento, durante un año en el Instituto Nacional de Neurología y Neurocirugía de la ciudad de México. La cirugía fue supervisada por el equipo canadiense, conformado por el Dr David Steven (neurocirujano de epilepsia) y el Dr. Jorge Burneo (neurólogoepileptologo)(4).

Posteriormente, otras cirugías fueron realizadas en el INCN, algunas de ellas supervisadas por el equipo UWO, pero la mayoría de manera independiente por el equipo del INCN, quien ya cuenta con más de 60 cirugías de epilepsia en su haber, desde el 2011. Su tasa de éxito es tan alta como la que se ve en centros experimentados en el hemisferio norte.

En el 2016, se realizó una lobectomía temporal en el hemisferio dominante, durante la cual se mantuvo al paciente despierto durante la mayor parte de la operación, con el objetivo de mapear el lenguaje. La parte de anestesiología fue llevada a cabo en conjunto por ambos equipos (de la Western University y del INCN).

Esta colaboración se sigue llevando a cabo, no solo centrándose en la investigación de pacientes con epilepsia refractaria, sino también en la educación continua del equipo del INCN en nuevos tratamientos de epilepsia, con énfasis en cirugía de epilepsia. Los siguientes pasos deseados, son el poder realizar evaluaciones de fase 2 , con implantación de electrodos intracraneales, pero lamentablemente, la inversión monetaria es mayor y dependiente del gobierno central.

Otros centros del Ministerio de Salud (MINSA) en Perú

El equipo canadiense también ha participado en conferencias sobre cirugía de epilepsia en otros centros en Lima, Trujillo y Cusco, con la idea de dar a conocer la existencia de la cirugía de epilepsia y orientar en el tratamiento de la epilepsia refractaria. Además, varios residentes de neurología de diferentes centros hospitalarios del Perú han rotado en el Hospital
Universitario afiliado a la Western University, donde se encuentra el programa de epilepsia.

\section{EsSALUD - Hospital Nacional Edgardo Rebagliati Martins (HNERM)}

En el año 2012, la neurocirujana Dra. Alicia Becerra aplico al fellowship de cirugía de epilepsia en la Western University. Luego de un año de entrenamiento, la Dra. Becerra regresó al Perú, y estableció conjuntamente con un equipo nuevo de neurólogos un centro incipiente de cirugía de epilepsia. Este centro incipiente inicialmente no contaba con una unidad de video-EEG, por lo que los video-EEG eran realizados en la práctica privada (realizados fuera del hospital). A pesar de los trámites que eran requeridos para obtener estos estudios de video-EEG, varias cirugías de epilepsia fueron llevadas a cabo en este centro, incluyendo cirugías con estimulación cortical directa y electrocorticografia intraoperatoria (13).

La reciente inauguración de una cama de videoEEG en el HNERM, permitirá incrementar el número de pacientes que podrán recibir cirugía de epilepsia. La última cirugía de epilepsia realizada en el hospital fue un caso de epilepsia temporal izquierda, fue la primera paciente registrada con el propio video EEG del hospital y fue operada con la presencia y supervisión del equipo de la UWO. El HNERM se espera sea un centro de referencia para la cirugía de epilepsia en el sistema de ESSALUD en el Perú.

\section{Puntos finales}

Es necesario que, en el Perú, existan más centros integrales de epilepsia, que incluyan cirugía de epilepsia, dado el alto número de pacientes refractarios a tratamiento médico. Es el objetivo de esta colaboración peruano canadiense el de ampliar lo logrado en el INCN y en el HNERM, así como llevar esta experiencia a otros centros hospitalarios de ESSALUD y del MINSA, y de ser posible en las principales provincias.

El éxito de la colaboración peruano canadiense (14) no solo reside en el apoyo financiero del ILAE y de otras entidades canadienses, así como la buena intención del equipo de la Western University, sino en el ímpetu, deseos de superación, de apoyo al paciente, y dedicación de un grupo de neurólogos y neurocirujanos peruanos. A pesar de las adversidades relacionadas a la burocracia de ambos sistemas (EsSalud/MINSA), a la competencia y muchas veces inadecuada relación 
entre neurólogos y neurocirujanos (la cual no es única en el Perú, sino en otras regiones del mundo), se pudieron establecer equipos multidisciplinarios de cirugía de epilepsia, que antes no existían en el Perú, y que hoy es un sueño hecho realidad.

\section{Correspondencia:}

Jorge G Burneo, MD, MSPH

Epilepsy Program

Department of Clinical Neurological Sciences

Schulich School of Medicine

Western University

University Hospital, LHSC

339 Windermere Rd

London, Ontario, Canada, N6A5A5

Correo electrónico: jburneo2@uwo.ca

\section{REFERENCIAS BIBLIOGRÁFICAS}

1. Moshe SL, Perucca E, Ryvlin P, Tomson T. Epilepsy: new advances. Lancet. 2015; 385: 884-98.

2. Burneo JG, Tellez-Zenteno J, Wiebe S. Understanding the burden of epilepsy in Latin America: a systematic review of its prevalence and incidence. Epilepsy Res. 2005;66:63-74.

3. Kwan P, Arzimanoglou A, Berg AT, Brodie MJ, Allen Hauser W, Mathern G, et al. Definition of drug resistant epilepsy: consensus proposal by the ad hoc Task Force of the ILAE Commission on Therapeutic Strategies. Epilepsia. 2010;51(6):1069-77. doi: 10.1111/j.1528-1167.2009.02397.x

4. Burneo JG, Delgado JC, Steven DA, Vasquez CM, Alonso-Vanegas MA, Cavazos JE, et al. A collaborative effort to establish a comprehensive epilepsy program in Peru. Epilepsy Behav. 2013;26:96-9.

5. Kwan P, Brodie MJ. Early identification of refractory epilepsy. N Engl J Med. 2000; 342: 314-9.

6. Pati S, Alexopoulos AV. Pharmacoresistant epilepsy: from pathogenesis to current and emerging therapies. Cleve Clin J Med. 2010;77:457-67.
7. Wiebe S, Blume WT, Girvin JP, Eliasziw M. Effectiveness, efficiency of surgery for temporal lobe epilepsy study G: A randomized, controlled trial of surgery for temporal-lobe epilepsy. N Engl J Med. 2001;345:311-8.

8. Engel J Jr, Wiebe S, French J, Sperling M, Williamson P, Spencer D, et al. Practice parameter: temporal lobe and localized neocortical resections for epilepsy: report of the Quality Standards Subcommittee of the American Academy of Neurology, in association with the American Epilepsy Society and the American Association of Neurological Surgeons. Neurology. 2003;60: 538-47.

9. Ryvlin P, Cross JH, Rheims S. Epilepsy surgery in children and adults. Lancet Neurol. 2014; 13:111426.

10. Parrent AG. Intracranial neurostimulation for epilepsy. The Canadian Journal of Neurological Sciences. 2012;39:S14-S9.

11. Burneo JG, Poon R, Kellett S, Snead OC. The utility of positron emission tomography in epilepsy. Can $\mathrm{J}$ Neurol Sci. 2015;42:360-71.

12. Burneo JG, Vezina W, Romsa J, Smith BJ, McLachlan RS. Evaluating the development of a SPECT protocol in a Canadian epilepsy unit. Can J Neurol Sci. 2007;34:225-9.

13. Barreto-Acevedo E, Becerra-Zegarra A, VillafuerteEspinoza MV, Llaja-Rojas V. Cirugía de epilepsia en patología dual abordada con estimulación cortical directa y electrocorticografía intraoperatoria. Primer caso en el Hospital Rebagliati. Rev Neuropsiquiatr. 2016;79:127-34.

14. Burneo JG, Steven DA, Arango M. Peru, Canada collaborate in epilepsy project. London: World Federation of Neurology; 2017. (Citado el 6 de junio del2017)Disponibleen:https://worldneurologyonline. com/article/peru-canada-collaborate-on-epilepsyproject/.

Recibido: 10/04/2017

Aceptado: 11/09/2017 